\title{
Interleukin-6-induced JAK2/STAT3 signaling pathway in endothelial cells is suppressed by hemodynamic flow
}

\author{
Chih-Wen Ni, Hsyue-Jen Hsieh, Yuen-Jen Chao and Danny Ling Wang \\ Am J Physiol Cell Physiol 287:771-780, 2004. First published May 19, 2004; \\ doi:10.1152/ajpcell.00532.2003
}

You might find this additional information useful...

This article cites 42 articles, 25 of which you can access free at:

http://ajpcell.physiology.org/cgi/content/full/287/3/C771\#BIBL

This article has been cited by 3 other HighWire hosted articles:

Mechanisms of induction of endothelial cell E-selectin expression by smooth muscle cells and its inhibition by shear stress

J.-J. Chiu, L.-J. Chen, C.-I Lee, P.-L. Lee, D.-Y. Lee, M.-C. Tsai, C.-W. Lin, S. Usami and S.

Chien

Blood, July 15, 2007; 110 (2): 519-528.

[Abstract] [Full Text] [PDF]

Laminar flow attenuates interferon-induced inflammatory responses in endothelial cells

Y.-C. Tsai, H.-J. Hsieh, F. Liao, C.-W. Ni, Y.-J. Chao, C.-Y. Hsieh and D. L. Wang

Cardiovasc Res, June 1, 2007; 74 (3): 497-505.

[Abstract] [Full Text] [PDF]

Thrombin, TNF-\{alpha\}, and LPS exert overlapping but nonidentical effects on gene expression in endothelial cells and vascular smooth muscle cells

S.-Q. Wu and W. C. Aird

Am J Physiol Heart Circ Physiol, August 1, 2005; 289 (2): H873-H885.

[Abstract] [Full Text] [PDF]

Updated information and services including high-resolution figures, can be found at:

http://ajpcell.physiology.org/cgi/content/full/287/3/C771

Additional material and information about $\boldsymbol{A J P}$ - Cell Physiology can be found at: http://www.the-aps.org/publications/ajpcell

This information is current as of November 16, 2008 .

AJP - Cell Physiology is dedicated to innovative approaches to the study of cell and molecular physiology. It is published 12 times a year (monthly) by the American Physiological Society, 9650 Rockville Pike, Bethesda MD 20814-3991. Copyright () 2005 by the American Physiological Society. ISSN: 0363-6143, ESSN: 1522-1563. Visit our website at http://www.the-aps.org/. 


\title{
Interleukin-6-induced JAK2/STAT3 signaling pathway in endothelial cells
}

\section{is suppressed by hemodynamic flow}

\author{
Chih-Wen Ni, Hsyue-Jen Hsieh, Yuen-Jen Chao, and Danny Ling Wang \\ Cardiovascular Division, Institute of Biomedical Sciences, Academia Sinica, Taipei, Taiwan 11529
}

Submitted 1 December 2003; accepted in final form 11 May 2004

\begin{abstract}
Ni, Chih-Wen, Hsyue-Jen Hsieh, Yuen-Jen Chao, and Danny Ling Wang. Interleukin-6-induced JAK2/STAT3 signaling pathway in endothelial cells is suppressed by hemodynamic flow. Am J Physiol Cell Physiol 287: C771-C780, 2004. First published May 19, 2004; 10.1152/ajpcell.00532.2003.-Endothelial cells (ECs) are constantly exposed to shear stress, the action of which triggers signaling pathways and cellular responses. During inflammation, cytokines such as IL-6 increase in plasma. In this study, we examined the effects of steady flow on IL-6-induced endothelial responses. ECs exposed to IL-6 exhibited STAT3 activation via phosphorylation of Tyr705. However, when ECs were subjected to shear stress, shear forcedependent suppression of IL-6-induced STAT3 phosphorylation was observed. IL- 6 treatment increased the phosphorylation of JAK2, an upstream activator of STAT3. Consistently, shear stress significantly reduced IL-6-induced JAK2 activation. Pretreatment of ECs with an inhibitor of MEK1 did not alter this suppression by shear stress, indicating that extracellular signal-regulated kinase (ERK1/2) was not involved. However, pretreatment of ECs with an endothelial nitric oxide synthase inhibitor (nitro-L-arginine methyl ester) attenuated this inhibitory effect of shear stress on STAT3 phosphorylation. Shear stress-treated ECs displayed decreased nuclear transmigration of STAT3 and reduced STAT3 binding to DNA. Intriguingly, ECs exposed to IL-6 entered the cell cycle, as evidenced by increasing $\mathrm{G}_{2} / \mathrm{M}$ phase, and shear stress to these ECs significantly reduced IL-6-induced cell cycle progression. STAT3-mediated IL-6-induced cell cycle was confirmed by the inhibition of the cell cycle in ECs infected with adenovirus carrying the inactive mutant of STAT3. Our study clearly shows that shear stress exerts its inhibitory regulation by suppressing the IL-6-induced JAK2/STAT3 signaling pathway and thus inhibits IL-6-induced EC proliferation. This shear force-dependent inhibition of IL-6-induced JAK2/STAT3 activation provides new insights into the vasoprotective effects of steady flow on ECs against cytokine-induced responses.
\end{abstract}

shear stress; nitric oxide; cell cycle

HEMODYNAMIC FORCES PLAY an important role in maintaining vascular integrity. Endothelial cells (ECs) lining the vascular wall are subject to hemodynamic forces, including flow-induced shear stress. Previous studies examined the mechanisms involved in transducing these mechanical forces into second messengers and changes in gene transcription (12). ECs exposed to shear stress activate the extracellular signal-regulated kinase $(E R K 1 / 2)$ and c-Jun $\mathrm{NH}_{2}$-terminal kinase (JNK) pathways $(9,23,26)$. Previous studies in our laboratory $(10,21,34)$ have shown that induction of the ERK pathway by shear stress plays a role in the activation of redox-sensitive genes such as monocyte chemotactic protein $(M C P-1)$, intercellular adhesion molecule-1 (ICAM-1), and c-fos. However, exposure of ECs to

Address for reprint requests and other correspondence: D. L. Wang, Cardiovascular Division, Institute of Biomedical Sciences, Academia Sinica, Taipei, Taiwan 11529 (E-mail: lingwang@ibms.sinica.edu.tw). shear stress was also shown to promote vasoprotective mechanisms. Berk and coworkers $(3,40)$ indicated that steady laminar flow decreases endothelial apoptosis and TNF-induced endothelial activation. This inhibition of TNF-induced signal transduction subsequently produces atheroprotective effects on the endothelium. Laminar shear stress also induces endothelial nitric oxide synthase (eNOS) activity and the expression of the eNOS gene, both of which are crucial in maintaining endothelial function $(4,9,11)$. Shear stress-induced release of nitric oxide (NO) plays an inhibitory role by attenuating the activation of these signaling pathways (28). Interestingly, increased expression of redox-sensitive genes mediated via the antioxidant response element in the promoter region of those genes was documented in ECs under steady flow $(8,13)$. Taken together, these findings suggest that laminar shear stress protects ECs from inflammation via the modulation of signaling pathways and transcriptional regulation.

IL-6, a key proinflammatory cytokine, is involved in a spectrum of diseases, including atherosclerosis, osteoporosis, arthritis, diabetes, and certain cancers. A recent study (27) indicated that chronic stress increases IL-6 level in plasma. IL-6 has been linked to cardiovascular diseases because of its role in enhancing the production of C-reactive protein, an important risk factor for myocardial infarction (27). Various cells, including ECs, are capable of secreting IL-6 upon stimulation of cytokines (16) or endotoxins (24) during inflammation. The released IL-6 preferentially activates STAT3 with the phosphorylation of Tyr705 via the JAK signaling pathway. STAT3 is a member of a family of functionally related STAT proteins that can be activated by various cytokines or growth factors and plays a key role in a variety of biological activities, including cell growth, differentiation, apoptosis, transformation, inflammation, and immune responses $(6,18)$. Although the role of IL-6 in tumor growth has been well studied (5), its impact on endothelial function has not been well defined. Earlier studies have shown that IL-6 increases endothelial permeability (31). IL-6-induced EC proliferation has also been reported $(17,20)$. Moreover, studies have demonstrated that IL-6 induces angiogenic activity in both ex vivo and in vivo assays $(17,20)$.

In this study, we show that JAK2/STAT3 pathway activation by IL-6 is rapidly suppressed upon the initiation of steady flow to ECs and that this suppression is shear force dependent. Furthermore, we show that shear stress retards progression of the IL-6-induced endothelial cell cycle. Thus laminar shear stress not only prevents endothelial inflammatory responses but also inhibits EC proliferation. This study provides a molecular

The costs of publication of this article were defrayed in part by the payment of page charges. The article must therefore be hereby marked "advertisement" in accordance with 18 U.S.C. Section 1734 solely to indicate this fact. 
A

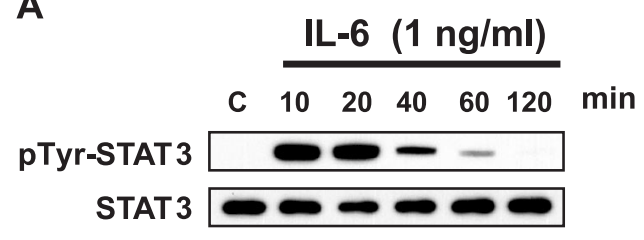

B

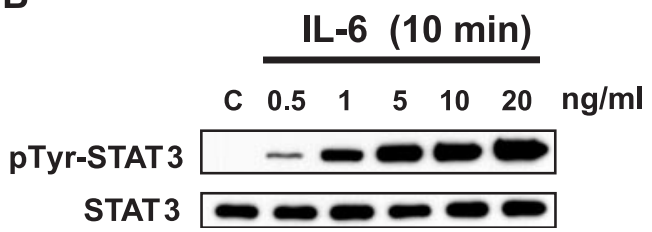

Fig. 1. IL-6 stimulates STAT3 tyrosine phosphorylation in endothelial cells (ECs). A: IL-6 induces tyrosine phosphorylation of STAT3. ECs were treated with $1 \mathrm{ng} / \mathrm{ml}$ IL-6 at different intervals as indicated. Cell lysates were subjected to Western blot analysis with anti-phospho-Tyr705 STAT3 (pTyr-STAT3) or anti-STAT3 (STAT3) as indicated. B: IL-6 increases STAT3 tyrosine phosphorylation in a concentration-dependent manner. ECs were treated with IL-6 at the indicated concentration for $10 \mathrm{~min}$, and the Tyr705 phosphorylation of STAT3 was examined. Results are representative of duplicate experiments with similar results. C, control.

basis for how shear stress protects ECs from cytokine-induced responses. The rapid inactivation of the JAK2/STAT3 pathway by laminar shear stress that leads to growth arrest provides a new mechanism for the vasoprotective effects exerted by hemodynamic flow on cytokine-induced vascular responses.

\section{MATERIALS AND METHODS}

Materials. The hemagglutinin antigen (HA)-tagged STAT3 (HASTAT3) and HA-STAT3F mutant (Tyr705Phe) overexpression plasmids were gifts from Dr. T. Hirano (Department of Molecular Oncology, Osaka University Medical School, Japan; Ref. 32). Adenovi- rus carrying STAT3 mutant (Ad-STAT3F) was a gift from Dr. C.-Y. F. Huang (National Health Research Institutes, Taipei, Taiwan). Recombinant human IL-6 was purchased from R\&D Systems (Minneapolis, MN). Anti-phospho-Tyr705 STAT3, anti-phospho-ERK, and anti-JAK2 were purchased from Upstate Biotechnology (Lake Placid, NY). Anti-STAT3 and anti-ERK1 were purchased from Transduction Laboratories (Lexington, KY). Anti-HA antibody was obtained from Covance Research Products (Berkeley, CA). Anti-phospho-JAK2 was purchased from Biosource International (Camarillo, $\mathrm{CA})$. Anti-nucleolin $(\alpha \mathrm{C} 23)$ and anti-tubulin were purchased from Santa Cruz Biotechnology (Santa Cruz, CA). PD-98059, U0126, AG490, and nitro-L-arginine methyl ester (L-NAME) were purchased from Calbiochem (Cambridge, MA). All other chemicals of reagent grade were obtained from Sigma (St. Louis, MO).

Cell culture and transfections. Bovine aortic ECs were cultured in DMEM supplemented with $10 \%$ fetal bovine serum (FBS), $100 \mathrm{U} / \mathrm{ml}$ penicillin, and $100 \mu \mathrm{g} / \mathrm{ml}$ streptomycin (Invitrogen/Life Technologies, Carlsbad, CA) as described (42). The culture medium was then changed to DMEM containing only $0.5 \%$ FBS overnight before steady flow experiments. Transient transfection was performed by using Lipofectamine (Invitrogen/Life Technologies, Carlsbad, CA) as described previously (42).

Shear stress experiments. ECs were exposed to shear stress in a parallel plate flow chamber as previously described (21). This continuous, well-defined flow of culture medium to the chamber was maintained with the use of a roller pump. The flow loop system was situated in an acrylic plastic enclosure maintained at $37^{\circ} \mathrm{C}$ by a heater. The $\mathrm{pH}$ of the medium was constantly maintained by continuous gassing of the medium reservoir with humidified $5 \% \mathrm{CO}_{2}$. The reservoir consisted of DMEM containing 0.5\% FBS.

Western blotting. For Western blot analysis, ECs were lysed with buffer containing $1 \%$ NP- $40,0.5 \%$ sodium deoxycholate, $0.1 \%$ SDS, and protease inhibitors. ECs were disrupted by repeated aspiration through a 21-gauge needle. After cellular debris were removed, the same amount of protein was subjected to SDS-PAGE. After being transferred to a nitrocellulose membrane, antigen was analyzed using specific antibodies. Antigen-antibody complexes were detected using
A

Fig. 2. Shear stress (SS) suppresses IL-6-induced STAT3 Tyr705 phosphorylation in a time- and shear force-dependent manner. A: ECs pretreated with 1 $\mathrm{ng} / \mathrm{ml} \mathrm{IL}-6$ for $10 \mathrm{~min}$ were maintained static or were exposed to SS $\left(16 \mathrm{dyn} / \mathrm{cm}^{2}\right)$ in medium containing IL-6 for an additional 10 or $30 \mathrm{~min}$. Cell lysates were subjected to Western blot analysis with antibody specific to pTyr-STAT3 or STAT3 as indicated. The intensity of Tyr705 phosphorylation was normalized to the level of STAT3 and is presented as mean \pm SE of 5 independent experiments. $* P<0.01$ vs. respective IL-6-treated ECs under static conditions. $B$ : ECs pretreated with $1 \mathrm{ng} / \mathrm{ml} \mathrm{IL}-6$ for $10 \mathrm{~min}$ were maintained static or exposed to SS in medium containing IL-6 for $10 \mathrm{~min}$ with increasing shear forces as indicated, and the phosphorylation state at Tyr705 of STAT3 was examined. STAT3 phosphorylation was normalized to the level of STAT3 and is presented as mean $\pm \mathrm{SE}$ from 3 independent experiments. ${ }^{*} P<$ 0.05 vs. static control ECs. $\# P<0.05$ vs. ECs under $\mathrm{SS}$ of $5 \mathrm{dyn} / \mathrm{cm}^{2} .+P<0.05$ vs. ECs under SS of 16 $\mathrm{dyn} / \mathrm{cm}^{2}$.
10 min IL-6 pretreatment
C $10301030 \mathrm{~min}$

$\square$ IL-6

点
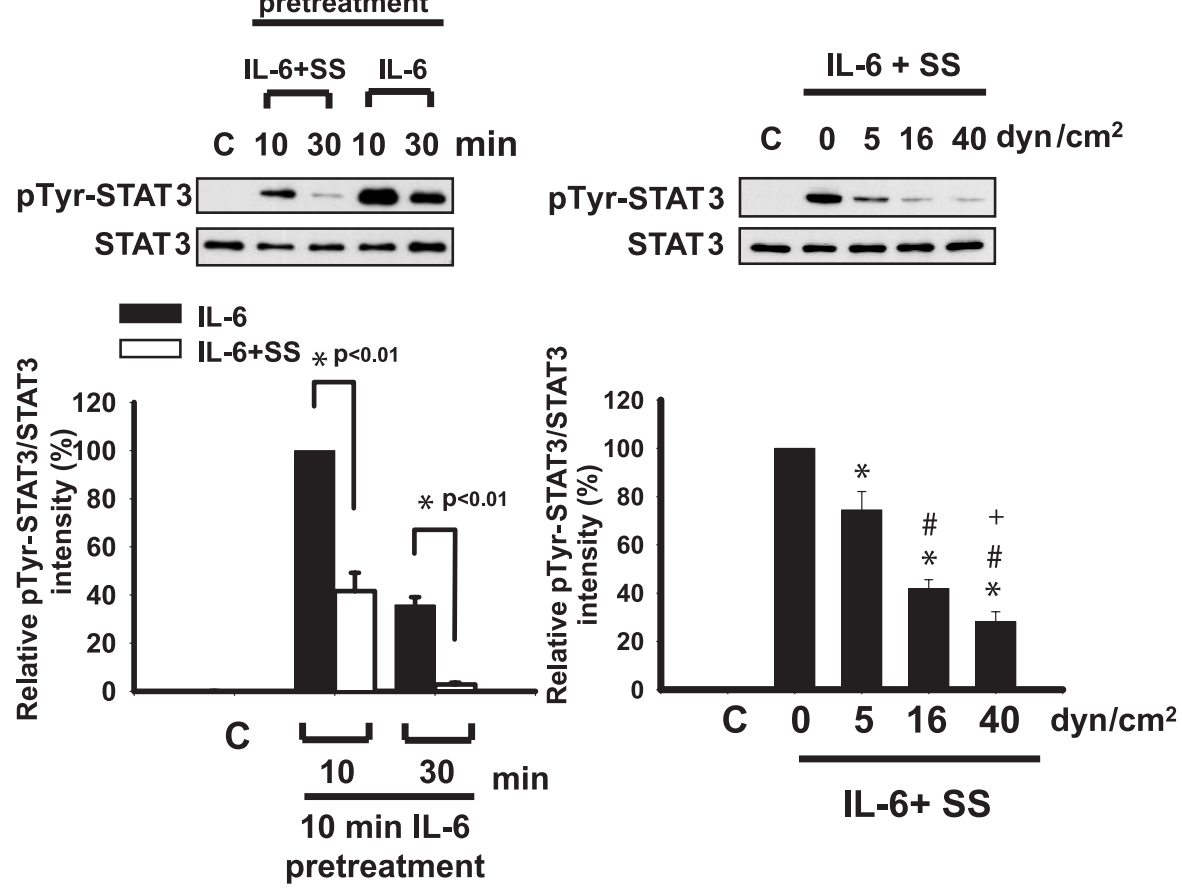

B 
A

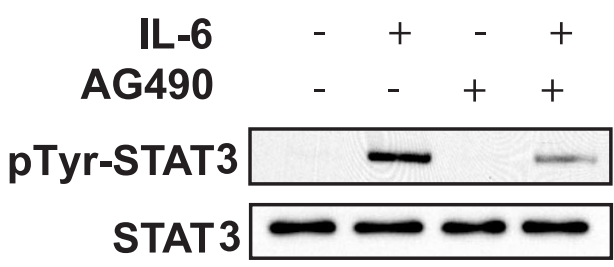

B

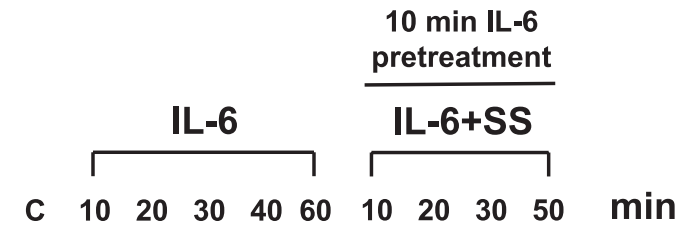

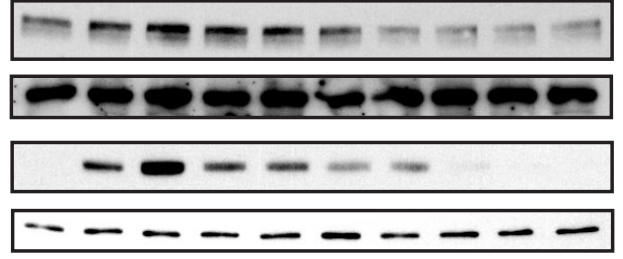

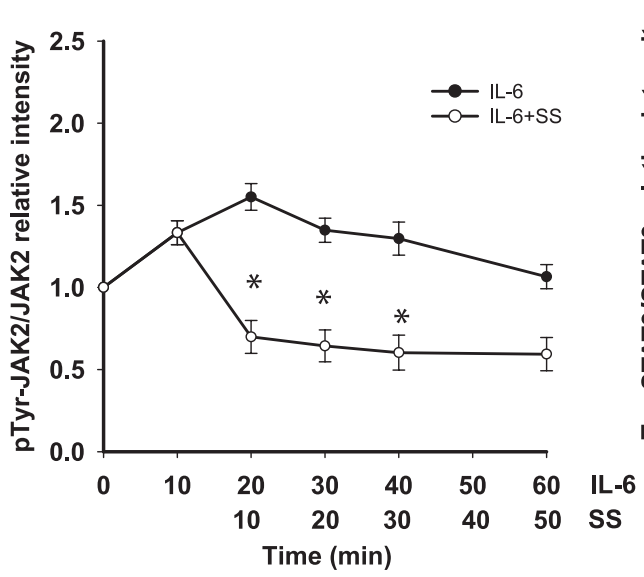

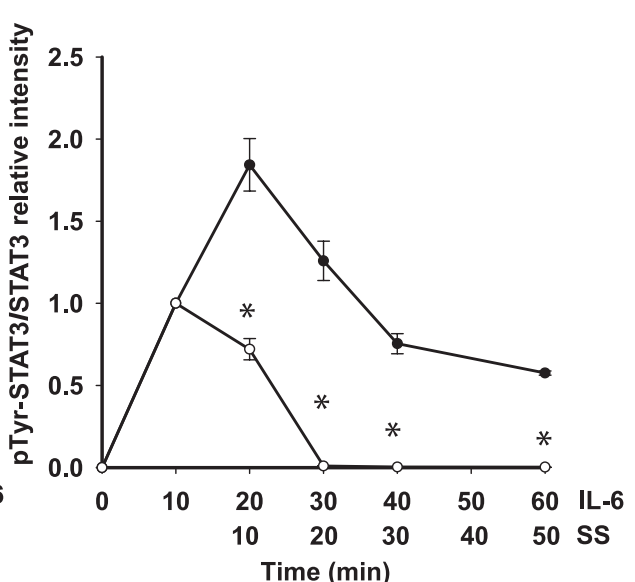

Fig. 3. JAK2 is involved in the inhibitory effect of SS on IL-6-induced STAT3 phosphorylation. A: ECs were pretreated with a JAK2 inhibitor (25 $\mu \mathrm{M}$ AG490) for $30 \mathrm{~min}$ and then maintained static or stimulated with IL-6 (1 ng/ml) for $20 \mathrm{~min}$. The phosphorylation of STAT3 was analyzed by Western blot using an antibody to pTyr-STAT3. $B$ : ECs pretreated with $1 \mathrm{ng} / \mathrm{ml} \mathrm{IL-6}$ for $10 \mathrm{~min}$ were either maintained static or exposed to SS (16 $\mathrm{dyn} / \mathrm{cm}^{2}$ ) in medium containing IL-6 for the intervals indicated. Cell lysates were subjected to Western blot analysis with an antibody to pTyr-STAT3 or phospho-Tyr705JAK2 (pTyr-JAK2). Antibodies to JAK2 or STAT3 were used to demonstrate that an equal amount of protein was applied to each lane. The intensity of Tyr705 phosphorylation was normalized to the level of STAT3. Results are presented as means $\pm \mathrm{SE}$ from at least 3 independent experiments. $* P<0.05$ vs. respective IL-6-treated ECs under static conditions horseradish peroxide-labeled rabbit anti-mouse or goat anti-rabbit IgG. Results were analyzed with an enhanced chemiluminescence detection system (ECL; Pierce).

Immunofluorescence microscopy. After treatment, ECs were fixed with $4 \%$ paraformaldehyde for $15 \mathrm{~min}$ and then washed with PBS containing $1 \%$ Triton X-100 for 15 min. ECs were blocked with $1 \%$ BSA and then treated with anti-HA for $1 \mathrm{~h}$ at room temperature. Secondary antibody labeled with FITC was used. HA-tagged wildtype or mutant STAT3 was transiently transfected into ECs and STAT3 intracellular localization was revealed by confocal laser scanning microscopy.

Nuclear protein extraction and electrophoretic mobility shift assay. Nuclear extracts from ECs after treatment were prepared as previously described (42). Nuclear proteins were analyzed by Western blot. The electrophoretic mobility shift assay (EMSA) was performed as previously described (42). End-labeled high-affinity Sis-inducible element (hSIE) (41) probe (0.5-2 ng) was added to the reaction mixture. Samples were then analyzed on a $5 \%$ nondenaturing polyacrylamide gel. For the supershift assay, anti-STAT3 or anti-STAT1 (Santa Cruz Biotechnology) was incubated with nuclear extracts before addition of the labeled hSIE probe.

Flow cytometry and cell cycle analysis. For cell cycle analysis, ECs were washed with cold PBS and then fixed and permeabilized with
$70 \%$ ethanol for $18 \mathrm{~h}$ at $-20^{\circ} \mathrm{C}$. ECs were then incubated with staining buffer $(0.1 \%$ Triton X-100, $0.2 \mathrm{mg} / \mathrm{ml}$ RNase A, and 20 $\mu \mathrm{g} / \mathrm{ml}$ propidium iodide) in the dark. Samples were filtered through $35-\mu \mathrm{m}$ nylon mesh, followed by flow cytometric analysis of DNA content using a Becton Dickinson flow cytometer (FACS Calibur). Cell cycle distribution was calculated using CellQuest software (Becton Dickinson).

Statistical analysis. Data are presented as means \pm SE. Statistical analysis was performed using one-way ANOVA. Differences were considered statistically significant at $P<0.05$.

\section{RESULTS}

IL-6-induced phosphorylation at Tyr705 of STAT3 is suppressed by shear stress. It is well documented that Tyr705 in STAT3 is phosphorylated after stimulation of cells by cytokines, including IL-6. To investigate whether IL-6 induces phosphorylation at Tyr705 of STAT3 in ECs, cells were treated with IL-6 at different time intervals. As shown in Fig. 1A, phosphorylation at Tyr705 of STAT3 rapidly increased within $10 \mathrm{~min}$ and then gradually returned to basal level after continuous exposure to IL-6 for $120 \mathrm{~min}$. This phosphorylation of 
A

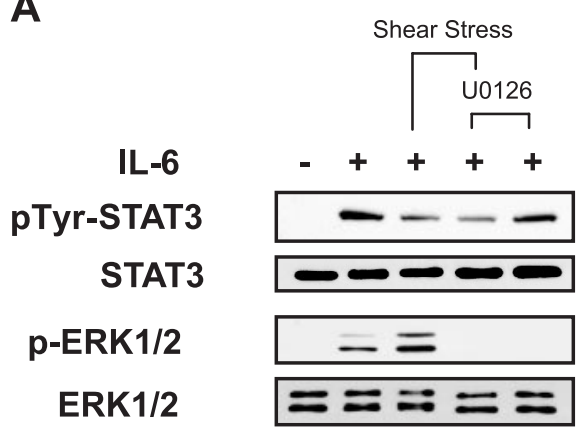

B

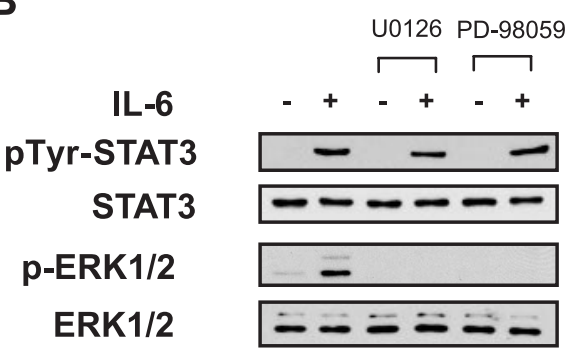

Fig. 4. Extracellular signal-regulated kinase (ERK1/2) is not involved in the inhibitory effect of SS on IL-6-induced STAT3 phosphorylation. A: ECs were pretreated with an inhibitor to MEK1 (10 $\mu \mathrm{M}$ U0126) for $30 \mathrm{~min}$ followed by $1 \mathrm{ng} / \mathrm{ml} \mathrm{IL-6} \mathrm{for} 10 \mathrm{~min}$ and then maintained static or exposed to SS for 10 min. The phosphorylation of STAT3 was analyzed by Western blot using antibody specific to pTyr-STAT3 or phospho-ERK1/2 (p-ERK1/2). B: ECs were pretreated with an inhibitor of MEK1 $(10 \mu \mathrm{M}$ U0126 or $30 \mu \mathrm{M}$ PD-98059) for $30 \mathrm{~min}$ and then maintained static or stimulated with $1 \mathrm{ng} / \mathrm{ml}$ IL-6 for $10 \mathrm{~min}$. The phosphorylation of STAT3 was analyzed by Western blot using an antibody to pTyr-STAT3 or p-ERK1/2. Similar results were obtained from 3 independent experiments.

Tyr705 was dependent on IL-6 concentration. The increased phosphorylation of Tyr705 was noticeable even in ECs treated with a low concentration of IL-6 $(0.5 \mathrm{ng} / \mathrm{ml})$ (Fig. 1B). Our results show that IL-6 triggers a rapid increase in phosphorylation of Tyr705 in STAT3.

Our previous study showed that shear stress induces Ser727 phosphorylation in STAT3 via the ERK1/2 pathway, and this Ser727 phosphorylation subsequently inhibits the serum-induced Tyr705 phosphorylation in STAT3 (33). To determine whether shear stress exerts a similar protective effect on IL-6-induced STAT3 activation, we treated ECs with IL-6, subjected the ECs to shear stress, and then examined the phosphorylation state of STAT3. In ECs pretreated with IL-6 $(1 \mathrm{ng} / \mathrm{ml})$ for $10 \mathrm{~min}$ followed by shear stress $\left(16 \mathrm{dyn} / \mathrm{cm}^{2}\right)$ in medium containing IL-6 (1 ng/ml) for $10 \mathrm{~min}$, IL-6-induced Tyr705 phosphorylation was significantly inhibited by $60 \%$ compared with IL-6-treated ECs under static conditions (Fig. $2 A$ ). Furthermore, when ECs were exposed to shear stress for 30 min, this IL-6-induced Tyr705 phosphorylation of STAT3 was further reduced to near basal level. More important, the inhibition of IL-6-induced Tyr705 phosphorylation by shear stress was shear force dependent (Fig. $2 B$ ); i.e., greater shear force applied to ECs resulted in decreased Tyr705 phosphorylation by IL-6.

IL-6-induced JAK2 phosphorylation is suppressed by shear stress. It is well documented that IL-6-induced STAT3 phosphorylation is mediated via JAK in many cell types $(6,18)$. ECs pretreated with a JAK2 inhibitor (AG490) showed greatly reduced IL-6-induced STAT3 phosphorylation (Fig. 3A). This is in agreement with previous results indicating that AG490 treatment downregulated the STAT3 pathway (14). We examined whether the shear stress inhibition of IL-6-induced STAT3 phosphorylation was mediated by JAK. IL-6 treatment increased JAK2 activation over the basal level of Tyr705 phosphorylation seen in control ECs (Fig. 3B). However, shear stress to ECs suppressed both IL-6-induced JAK2 phosphorylation and STAT3 phosphorylation. Although IL-6 treatment of ECs also induced JAK1 phosphorylation, shear stress on these ECs did not show a significant effect on IL-6-induced JAK1 phosphorylation (data not shown). Thus decreased JAK2 activation may account, at least partially, for the inhibitory effect of shear stress on IL-6-induced STAT3 activation.

ERK is not involved in STAT3 dephosphorylation caused by shear stress. Our previous work demonstrated that shear stress attenuates serum-induced STAT3 Tyr705 phosphorylation via ERK activation (33). Our current research shows that shear stress suppresses IL-6-induced Tyr705 phosphorylation of STAT3. We next investigated whether the ERK pathway is also involved in STAT3 dephosphorylation that occurs upon exposure to shear stress in the presence of IL-6. ECs were pretreated with an inhibitor of MEK1 (U0126) and then subjected to shear stress in the presence of IL-6. Surprisingly, the diminished level of STAT3 phosphorylation by shear stress was not restored even if ERK phosphorylation was inhibited substantially (Fig. 4A). Further study of IL-6-induced STAT3 phosphorylation indicated that it was independent of ERK activation because ECs pretreated with a MEK1 inhibitor (U0126 or PD-98059) did not affect IL-6-induced Tyr705 phosphorylation (Fig. 4B). Thus ERK activation is not involved in this STAT3 dephosphorylation induced by shear

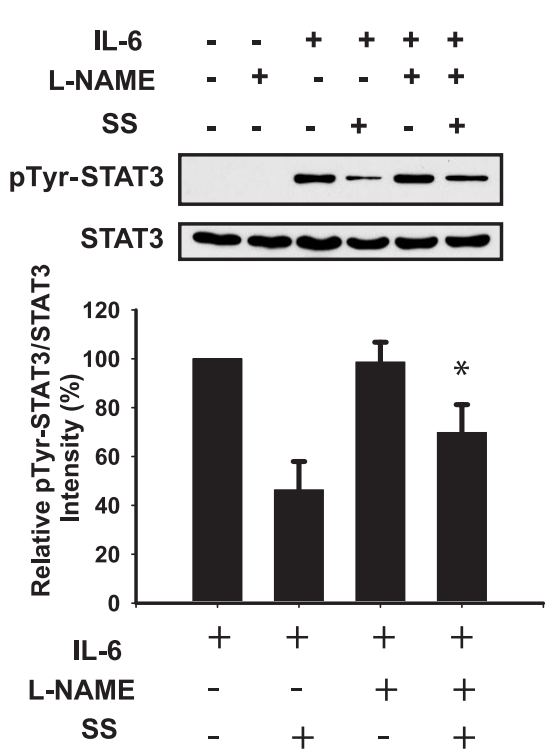

Fig. 5. Nitric oxide (NO) is involved in the inhibitory effect of SS on IL-6-induced STAT3 phosphorylation. A: ECs were pretreated with an inhibitor to endothelial nitric oxide synthase [100 $\mu \mathrm{M}$ nitro-L-arginine methyl ester (L-NAME)] for $30 \mathrm{~min}$ followed by $1 \mathrm{ng} / \mathrm{ml} \mathrm{IL-6} \mathrm{for} 10 \mathrm{~min}$ and then maintained static or exposed to SS for $10 \mathrm{~min}$. The phosphorylation of STAT3 was analyzed by Western blot using antibody specific to either pTyr-STAT3 or STAT3. $B$ : STAT3 phosphorylation was normalized to the level of STAT3 and is presented as mean $\pm \mathrm{SE}$ from 3 independent experiments. ${ }^{*} P<0.05$ vs. ECs under SS in the presence of L-NAME. 


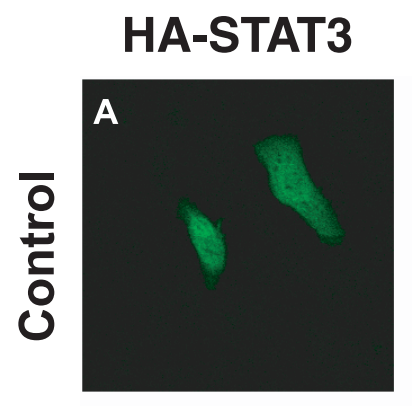

\section{HA-STAT3F}
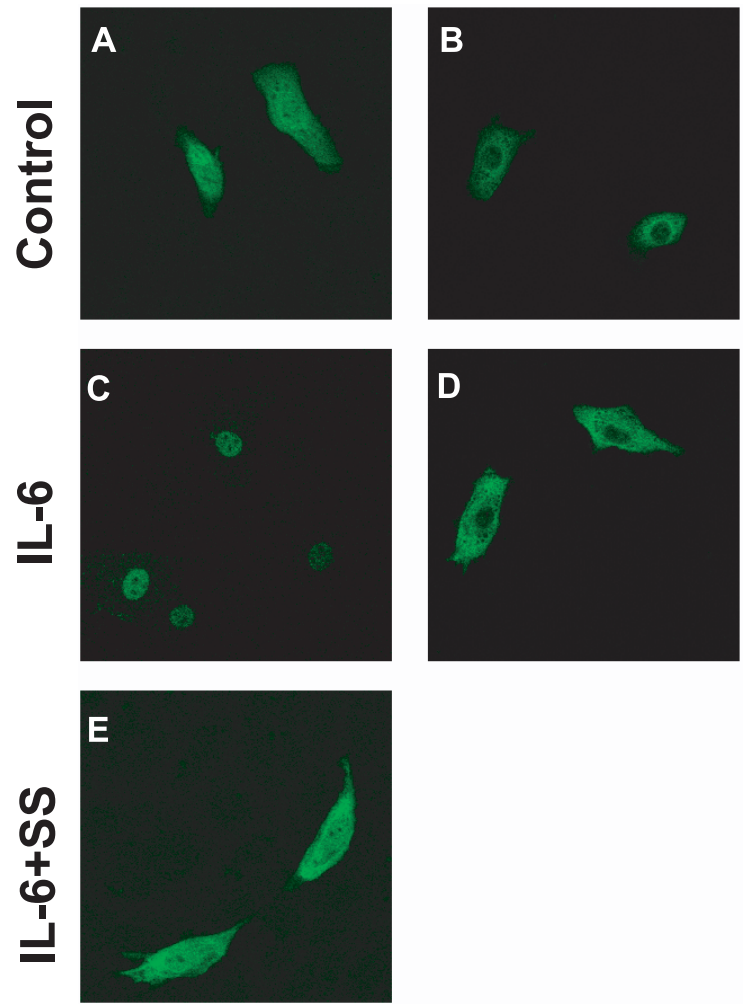

Fig. 6. Tyr705 phosphorylation is required for STAT3 nuclear translocation. ECs transiently transfected with hemagglutinin antigen (HA)-tagged STAT3 (HA-STAT3) $(A, C$, and $E$ ) or HA-tagged STAT3 point mutant containing a phenylalanine substitution at position 705 (HA-STAT3F) $(B$ and $D$ ) were incubated with $(C$ and $D)$ or without $(A$ and $B)$ IL-6 $(20 \mathrm{ng} / \mathrm{ml})$ for $15 \mathrm{~min}$. ECs overexpressing HA-STAT3 were subjected to SS with medium containing IL-6 for $15 \mathrm{~min}(E)$. ECs were fixed, permeabilized, and incubated with anti-HA, followed by incubation with FITC-conjugated secondary antibody. ECs were visualized by confocal laser scanning microscopy.

stress in IL-6-treated ECs. This result suggests that shear stress suppresses IL-6-induced STAT3 activation via a different signaling mechanism.

Shear stress-induced STAT3 dephosphorylation is mediated by NO. Shear stress has been shown to induce the activity of eNOS, which is crucial in maintaining endothelial function (4, $9,11)$. The released NO can exert its inhibitory role by attenuating signaling pathways and gene expression (28). We demonstrated earlier that endothelial NO acted as a negative regulator in endothelial responses to hemodynamic forces by suppressing ERK activity and early growth response-1 expression (9). To assess the role of NO in this shear stress-induced inhibitory effect on STAT3 signaling, ECs were pretreated with a specific inhibitor to eNOS (100 $\mu \mathrm{m}$ L-NAME) and then stimulated with IL-6 under shear stress. As shown in Fig. 5, the suppression of IL-6-induced Tyr705 phosphorylation of STAT3 by shear stress is significantly attenuated in the presence of L-NAME. Our results demonstrate that NO is involved in the inhibitory effect of shear stress on STAT3 signaling. Shear stress-induced NO may play a role by modulating endothelial responses to IL-6.

IL-6-induced STAT3 transmigration into the nucleus is attenuated by shear stress. Phosphorylation of Tyr705 is required for STAT3/3 dimer formation and for nuclear translo- cation $(6,18)$. To investigate whether the suppression of Tyr705 phosphorylation in STAT3 by shear stress prevents STAT3 from transmigrating into the nucleus, wild-type STAT3 (HA-STAT3) and a STAT3 point mutant containing a Phe substitution at position 705 (HA-STAT3F) were compared in translocation assays. ECs were transiently transfected with HA-STAT3 or HA-STAT3F, and STAT3 translocation was analyzed by confocal microscopy after staining with anti-HA. HA-STAT3 rapidly translocated into the nucleus of ECs exposed to IL-6 (Fig. 6, $A$ and $C$ ). In contrast, there was no detectable translocation of HA-STAT3F into the nucleus in ECs from either untreated controls or ECs exposed to IL-6 (Fig. 6, B and D), indicating that Tyr705 phosphorylation is required for nuclear translocation of STAT3. More important, when ECs were exposed to shear stress, IL-6-induced HASTAT3 nuclear translocation was significantly reduced (Fig.

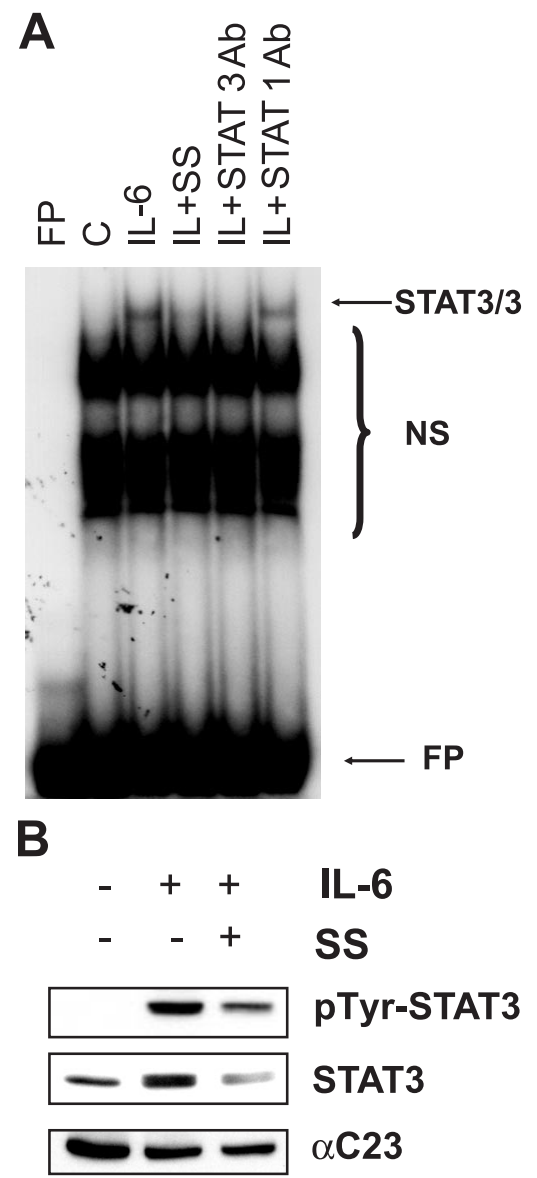

Fig. 7. SS inhibits nuclear transmigration and DNA binding of STAT3. A: ECs were maintained under static conditions (control, C) or treated with $20 \mathrm{ng} / \mathrm{ml}$ IL-6 for $15 \mathrm{~min}$ (IL-6). ECs were exposed to SS (16 dyn/ $\mathrm{cm}^{2}$ ) for $15 \mathrm{~min}$ in the presence of IL-6 (IL + SS). Nuclear extracts were prepared and analyzed by EMSA using a ${ }^{32} \mathrm{P}$-labeled high-affinity Sis-inducible element (hSIE) oligonucleotide probe. The specificity of the retarded complex (STAT3/3) was assessed by preincubating the nuclear extracts with an antibody to STAT3 $(\mathrm{IL}+\mathrm{Stat} 3 \mathrm{Ab})$ or STAT1 (IL+Stat1Ab). Results are representative of 3 independent experiments that yielded similar results. NS, nonspecific; FP, free probe. $B$ : ECs treated with $20 \mathrm{ng} / \mathrm{ml} \mathrm{IL-6}$ for $15 \mathrm{~min}$ were kept static or exposed to SS with medium containing identical IL-6 concentrations. Nuclear extracts were prepared and subjected to Western blot analysis with an antibody to pTyr-STAT3 or STAT3 as indicated. Anti-nucleolin $(\alpha \mathrm{C} 23)$ was used as a nuclear marker. Results are representative of 3 independent experiments that yielded similar results. 
A B

Control
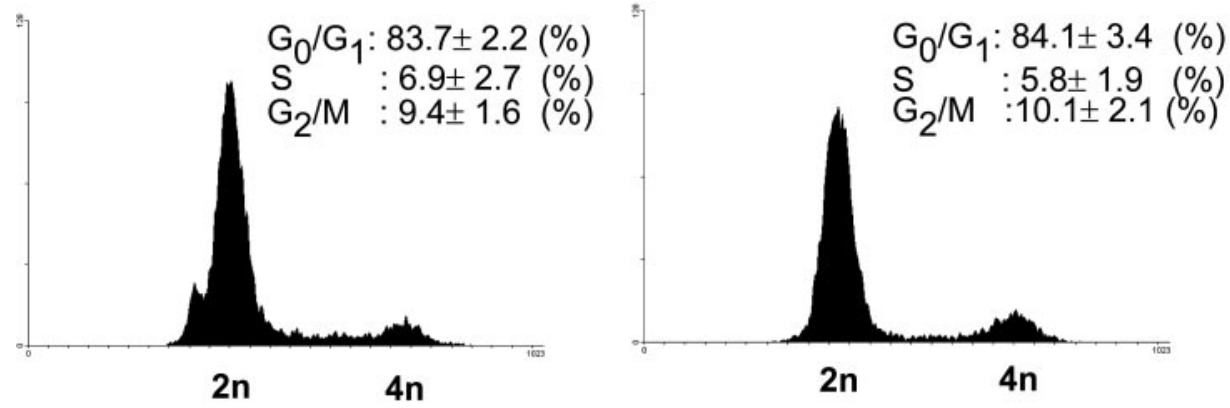

Fig. 8. SS inhibits IL-6-induced cell cycle progression. Cell cycle analysis was performed by flow cytometry using propidium iodide (PI) staining. A: ECs were starved overnight and then maintained under static conditions (control) or subjected to SS (16 $\mathrm{dyn} / \mathrm{cm}^{2}$ ) for $6 \mathrm{~h}$. ECs were fixed and prepared for flow cytometry. Cell cycle distribution was analyzed by PI DNA staining. $B$ : ECs were starved overnight and then cultured with or without $20 \mathrm{ng} / \mathrm{ml} \mathrm{IL-6} \mathrm{for} 6 \mathrm{~h}$. ECs were exposed to SS for $6 \mathrm{~h}$ in the presence of IL-6 (IL-6+SS). Cell cycle distribution was then analyzed.
SS

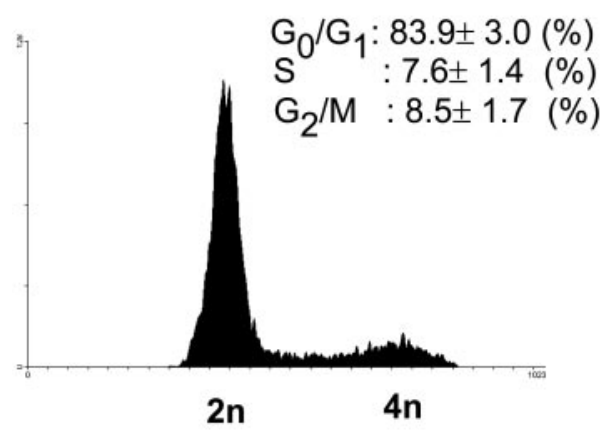

IL-6

\section{Control}

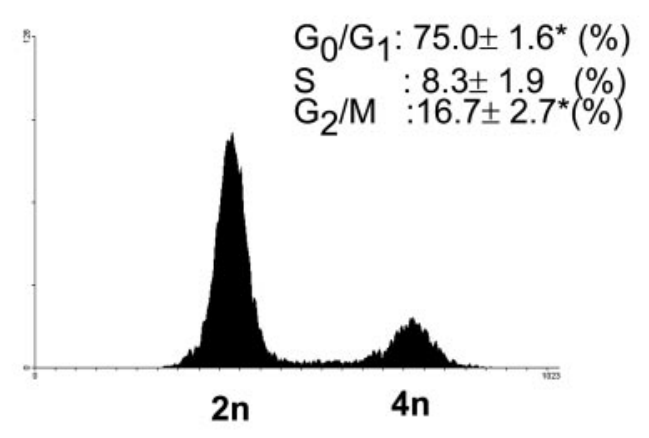

IL-6 + SS

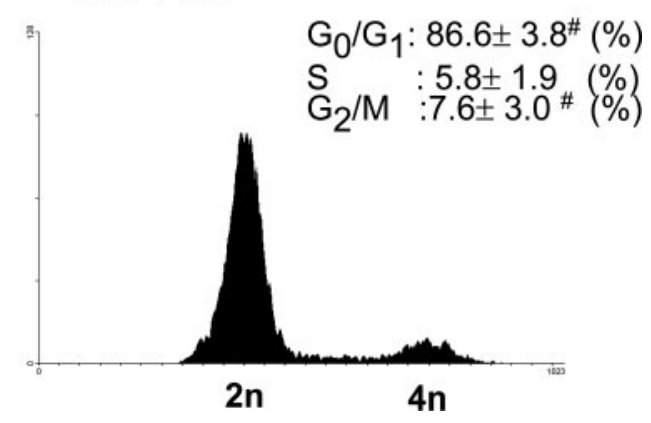

$6 E)$. These results clearly demonstrate that phosphorylation of Tyr705 is crucial for the nuclear transmigration of STAT3 and that shear stress attenuates this STAT3 activation, resulting in a decrease in its nuclear translocation. Subsequent to STAT3 activation and nuclear translocation, the STAT3/3 dimer binds to its specific DNA sequence and induces gene expression (6, 18). Because shear stress suppressed IL-6-induced STAT3 activity, we examined whether shear stress reduced STAT3 binding to DNA. EMSA was performed using the ${ }^{32} \mathrm{P}$-labeled oligonucleotide probe hSIE (Fig. 7A). The STAT3-DNA complex (STAT3/3) was clearly observed in IL-6-treated ECs (Fig. $7 A$ ). In contrast, STAT3/3 was only minimally detected in those IL-6-treated ECs subjected to shear stress (Fig. 7A). EMSA specificity was confirmed using nuclear protein extract preincubated with STAT3 antibody (Fig. 7A). The specificity of STAT3 was further substantiated by STAT3 transmigration into the nucleus. As shown in Fig. 7B, shear stress on IL-6treated ECs reduced STAT3 transmigration to the nucleus. This result is in agreement with the decreased Tyr705 phos- phorylation and reduced DNA-binding activity of STAT3. All of these data indicate that STAT3 activation by IL-6 is significantly inhibited by shear stress via the suppression of Tyr705 phosphorylation and a concomitant decrease in STAT3-mediated transcriptional activity.

IL-6-induced cell cycle progression inhibited by shear stress is mediated by STAT3. Previous studies demonstrated that IL-6 induces EC proliferation $(17,20)$. Cell cycle progression was examined by performing flow cytometric analysis of DNA content in IL-6-treated ECs exposed to shear stress. ECs were starved and then exposed to IL- 6 followed by shear stress for $6 \mathrm{~h}$. ECs primarily remained quiescent $\left(\mathrm{G}_{0} / \mathrm{G}_{1}\right)$ after starvation (Fig. 8A). Under the starvation condition, there was no significant difference in cell cycle status between control and sheared ECs. As expected, IL-6 treatment of ECs increased DNA synthesis and shifted the population to the $G_{2} / M$ phase (Fig. 8B). In contrast, IL-6-treated ECs subjected to shear stress remained predominantly in $\mathrm{G}_{0} / \mathrm{G}_{1}$. These results indicate that shear stress on ECs inhibits IL-6-stimulated cell cycle 
A

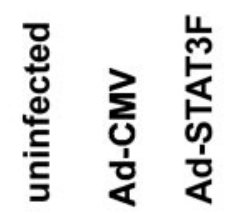

STAT3

tubulin

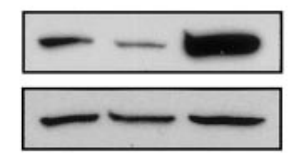

B

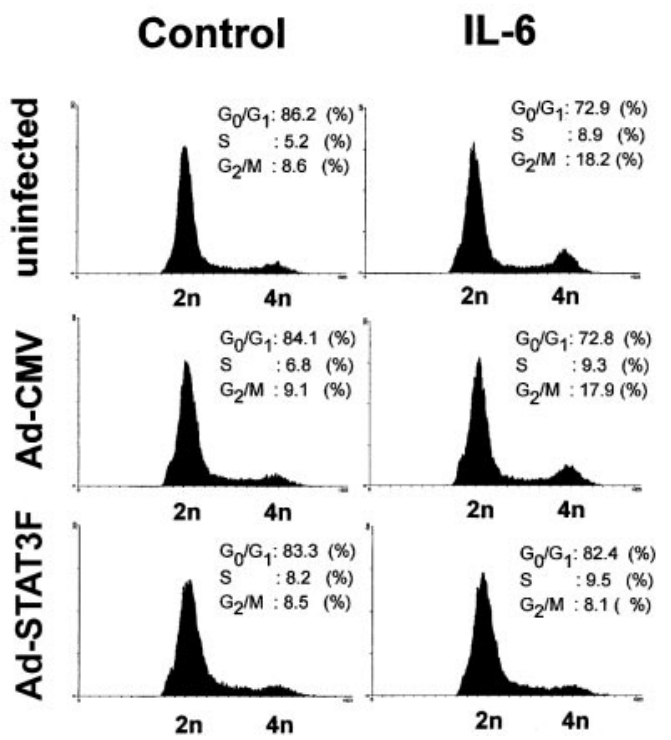

Fig. 9. IL-6-induced cell cycle progression is mediated by STAT3. A: ECs infected with Ad-STAT3F increased total STAT3 protein. ECs were either maintained in the unstimulated condition (uninfected) or infected with an empty vector (Ad-CMV) or adenovirus carrying a STAT3 Tyr705 mutant (Ad-STAT3F). B: ECs were infected with empty vector or Ad-STAT3F in serum-free DMEM. Serum was added after $2 \mathrm{~h}$ of incubation, and the cultures were maintained for an additional $22 \mathrm{~h}$. ECs were then starved in DMEM containing $0.5 \%$ FBS overnight. ECs were treated with $20 \mathrm{ng} / \mathrm{ml} \mathrm{IL-6}$ for $6 \mathrm{~h}$. ECs were fixed and analyzed by flow cytometry. Cell cycle distribution was analyzed by PI staining.

progression. To further confirm whether IL-6-induced cell cycle progression was mediated by STAT3, ECs were infected with adenovirus carrying a dominant negative mutant of STAT3 (Ad-STAT3F) (Fig. 9A). IL-6 treatment significantly induced cell cycle progression in ECs infected with empty viral vector (Ad-CMV) or in uninfected control ECs. In contrast, Ad-STAT3F-infected ECs showed no response to IL-6 (Fig. 9B). These results confirm that IL-6 induction of the endothelial cell cycle is mediated by STAT3 and that shear stress to ECs inhibits this STAT3-mediated cell cycle progression. Taken together, our results clearly show that laminar shear stress acts as a negative regulator by inhibiting IL-6-induced STAT3 activation, resulting in suppression of IL-6-mediated EC proliferation.

\section{DISCUSSION}

During inflammation, IL-6 is produced and secreted by various cells, including ECs (16). The pathological effects of IL-6 on vessel walls are not clear. Vascular ECs are constantly subjected to shear stress. The present study shows that IL-6-induced STAT3 activation is rapidly suppressed upon the initiation of steady flow to ECs. This rapid inactivation of STAT3 represents a vasoprotective mechanism via shear stress. We have shown that IL-6 induces dose-dependent STAT3 activation, although shear stress to ECs rapidly suppressed this activation. Several lines of evidence support this notion. First, the shear stress that inhibited IL-6-induced Tyr705 phosphorylation of STAT3 was time dependent. Second, this inhibition by shear stress was shear force dependent, because we observed that increased shear force progressively suppressed IL-6-induced Tyr705 phosphorylation. Third, shear stress inhibited the IL-6-induced activation of JAK2, an upstream activator of STAT3. This inhibitory effect by shear stress on IL-6induced STAT3 activation was further substantiated by EMSA results that showed decreased STAT3 binding to its corresponding oligonucleotide, a phenomenon also supported by a decrease in STAT3 transmigration into nuclei in IL-6-treated ECs exposed to shear stress.

It is well established that IL-6 binds to membrane receptor complexes and leads to the activation of the JAK/STAT3 pathway. Interestingly, in addition to JAK/STAT3 activation, IL-6 induced ERK activation (Fig. 3), which is crucial for cell survival and proliferation $(7,35,37)$. Earlier studies, including ours, showed that shear stress activates the ERK pathway $(25,26,29,33)$. Our more recent studies demonstrated that shear stress to ECs inhibited serum-induced Tyr705 phosphorylation of STAT3 and that inhibition is shear force dependent (33). Shear stress activated ERK1/2 and subsequently induced phosphorylation of Ser727. This ERK-dependent Ser727 phosphorylation negatively regulated Tyr705 phosphorylation, a phenomenon similar to earlier findings that phorbol 12-myristate 13-acetate (PMA) blocks IL-6-induced STAT3 activation via the ERK signaling pathway (38). The current study, however, suggests that ERK activation is not responsible for the shear stressinduced inhibitory effect on IL-6-activated STAT3 phosphorylation, because inhibition of the ERK signaling pathway does not alter the suppression effect of shear stress on STAT3 phosphorylation (Fig. 3A).

The STAT3 activation is a highly regulated process that includes a negative feedback mechanism involving members of the suppressors of cytokine signaling (SOCS) protein family $(6,39)$. However, the induction of SOCS is a transcriptional event. In contrast, our present data indicate that the immediate suppression of IL-6-induced STAT3 activation by shear stress is likely the consequence of a direct signaling event, although the details of this suppressive mechanism are not clear. ECs treated with AG490, a specific inhibitor of JAK2 (an upstream activator of STAT3), resulted in the inhibition on IL-6-induced Tyr705 phosphorylation of STAT3 (Fig. 3), indicating that JAK2 acts upstream of STAT3 activation. Shear stress on ECs also reduces IL-6-induced JAK2 activation, suggesting that JAK2 may be a candidate mediator of STAT3 suppression. Alternatively, shear stress may activate specific phosphatases that subsequently inhibit IL-6-induced JAK2/ STAT3 activation. This possibility is supported by findings 
Fig. 10. Inhibitory effect of SS on IL-6-induced STAT3 activation. IL-6 induces the tyrosine phosphorylation $(\mathrm{P})$ of JAK2 and STAT3, which in turn leads to the nuclear transmigration of STAT3 and an induction of cell cycle progression. SS induces signaling events, including the activation of the ERK1/2 and phosphatidylinositol 3-kinase (PI3K)/Akt pathways. ERK1/2 activation contributes to the phosphorylation of Ser727 (33). This ERK1/2 activation, however, is not involved in the inhibitory effect of SS. The increased NO production decreases activation of STAT3. Protein tyrosine phosphatase(s) [PTP(s)] may play an important role by modulating the phosphorylation of STAT3. The detailed mechanisms remain to be defined. eNOS, endothelial nitric oxide synthase; gp130, glycoprotein 130 .

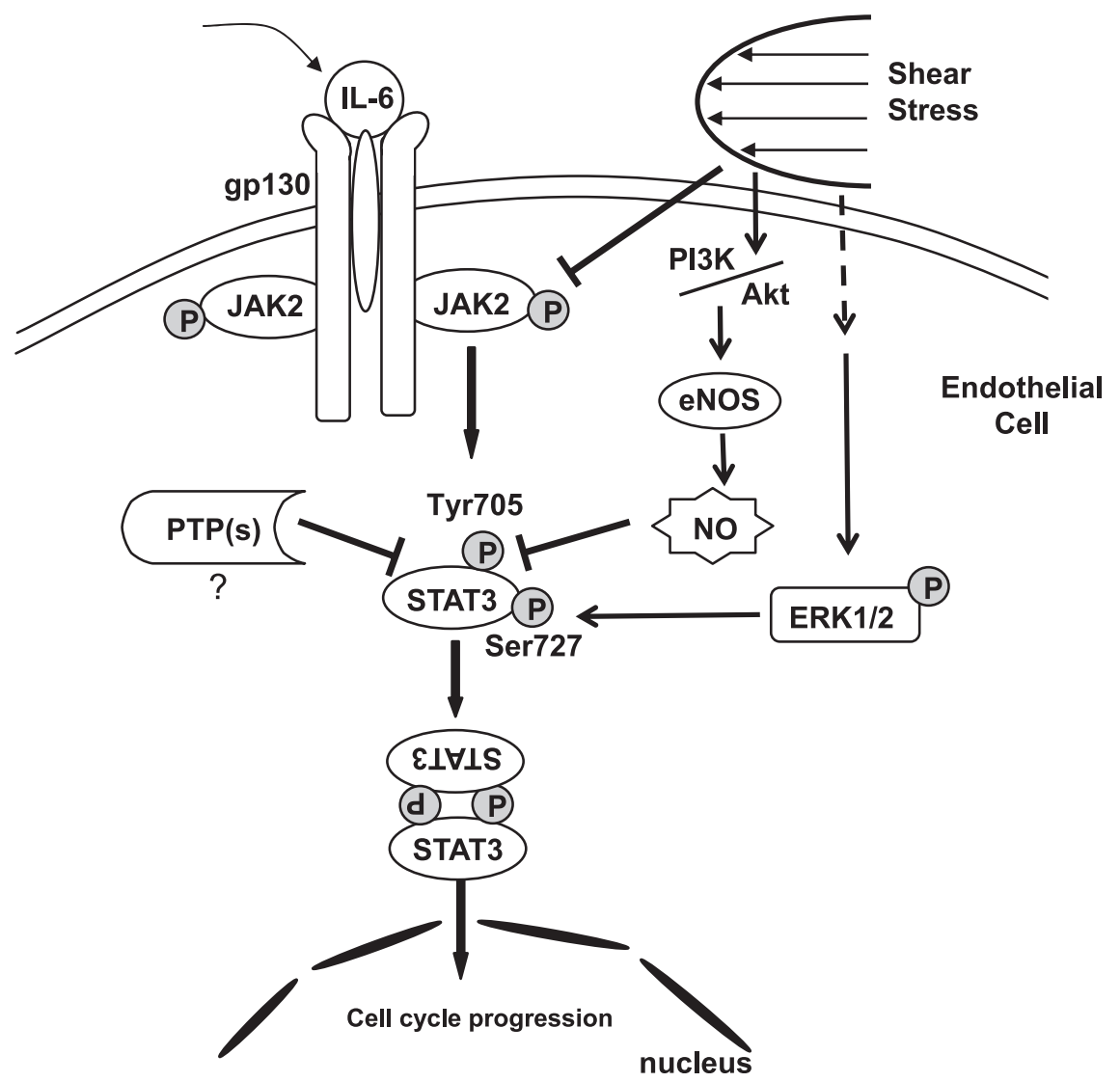

that STAT signaling is suppressed by counterinhibition of tyrosine kinase activity by a tyrosine phosphatase (22) and that shear stress suppression of IL-6-induced Tyr705 phosphorylation in STAT3 is significantly attenuated when ECs are pretreated with the tyrosine phosphatase inhibitor sodium orthovanadate (Ni CW and Wang DL, unpublished observation).

IL-6 reportedly induces EC proliferation $(17,20)$. IL-6activated STAT3 is involved in the induction of cyclin D1, c-myc, and Bcl-xL, each of which participates in the regulation of the cell cycle and/or the induction of apoptosis (2, 5,19 ). Additional evidence shows that STAT3 activation by growth factors or cytokines is involved in cell growth, particularly during tumor progression $(6,18)$, and that inactive STAT3 may hamper EC proliferation and integrity. Interestingly, we have demonstrated that IL-6-induced cell cycle progression is inhibited in ECs exposed to shear stress. The role of IL-6 activation of STAT3 in the induction of cell cycle entry is supported by the observation that ECs infected with adenovirus carrying an inactive STAT3 mutant had a reduced proliferative index. Our results strongly suggest that IL-6-induced STAT3 activation plays an important role in EC proliferation. High shear stress has been shown to inhibit EC proliferation $(1,15,30)$. Lin et al. (30) indicated that laminar shear stress to ECs inhibits the endothelial cell cycle by inducing the expression of the growth arrest protein GADD45 and $\mathrm{p} 21^{\text {cip } 1}$. Our current study presents another mechanism governing growth arrest by shear stress on IL-6-activated ECs that includes the phosphorylation-dependent regulation of STAT3 activation.

Multiple recurrent inflammatory events contribute to the initiation and progression of atherosclerosis $(3,36)$. It is possible that IL- 6 produced during inflammation activates JAK2/STAT3 and initiates EC proliferation and inflammatory response. Our present data, however, show that laminar shear stress exerts an atheroprotective effect by attenuating the JAK2/STAT3 signaling process. Although the detailed inhibitory mechanisms remain to be elucidated, the ERK signaling pathway is not involved, but NO appears to play a role by suppressing STAT3 activation. Protein tyrosine phosphatase may also contribute to this shear stress-induced STAT3 dephosphorylation. Figure 10 shows the inhibitory effect of shear stress on STAT3 activation and the potential regulatory mechanisms involved. This shear force-dependent inhibition of IL-6-stimulated JAK2/STAT3 activation provides a molecular basis for how the shear stress protects ECs from cytokine-induced responses, including cell division. This represents a new negative regulatory mechanism imposed by laminar shear stress on ECs. Our findings are of physiological significance because it is well known that atherosclerotic lesions develop in areas where low shear or nonlaminar flow is encountered, and these areas are usually associated with elevated EC proliferation. Our results provide new insight into atheroresistant mechanisms in the straight segments of the arterial tree where high shear stress and low growth rate normally occur. 


\section{GRANTS}

This work was supported in part by the National Science Council, Taiwan (NSC 92-2320-B001-020) and by the Department of Education Program for Promoting Academic Excellence of Universities, Taiwan (91-B-FA09-2-4).

Present address of H.-J. Hsieh: Dept. of Chemical Engineering, National Taiwan University, Taipei, Taiwan 106.

\section{REFERENCES}

1. Akimoto S, Mitsumata M, Sasaguri T, and Yoshida Y. Laminar shear stress inhibits vascular endothelial cell proliferation by inducing cyclindependent kinase inhibitor p21(Sdi1/Cip1/Waf1). Circ Res 86: 185-190, 2000 .

2. Benekli M, Xia Z, Donohue KA, Ford LA, Pixley LA, Baer MR, Baumann H, and Wetzler M. Constitutive activity of signal transducer and activator of transcription 3 protein in acute myeloid leukemia blasts is associated with short disease-free survival. Blood 99: 252-257, 2002.

3. Berk BC, Abe JI, Min W, Surapisitchat J, and Yan C. Endothelial atheroprotective and anti-inflammatory mechanisms. Ann NY Acad Sci 947: 93-111, 2001.

4. Boo YC, Sorescu G, Boyd N, Shiojima I, Walsh K, Du J, and Jo H. Shear stress stimulates phosphorylation of endothelial nitric-oxide synthase at $\operatorname{Ser}^{1179}$ by Akt-independent mechanisms: role of protein kinase A. J Biol Chem 277: 3388-3396, 2002.

5. Bowman T, Garcia R, Turkson J, and Jove R. STATs in oncogenesis. Oncogene 19: 2474-2488, 2000.

6. Bromberg JF. Activation of STAT proteins and growth control. Bioessays 23: 161-169, 2001

7. Bullard LE, Qi X, and Penn JS. Role for extracellular signal-responsive kinase-1 and -2 in retinal angiogenesis. Invest Ophthalmol Vis Sci 44: 1722-1731, 2003.

8. Chen XL, Varner SE, Rao AS, Grey JY, Thomas S, Cook CK, Wasserman MA, Medford RM, Jaiswal AK, and Kunsch C. Laminar flow induction of antioxidant response element-mediated genes in endothelial cells: a novel anti-inflammatory mechanism. J Biol Chem 278: 703-711, 2003.

9. Chiu JJ, Wung BS, Hsieh HJ, Lo LW, and Wang DL. Nitric oxide regulates shear stress-induced early growth response-1: expression via the extracellular signal-regulated kinase pathway in endothelial cells. Circ Res 85: 238-246, 1999

10. Chiu JJ, Wung BS, Shyy JY, Hsieh HJ, and Wang DL. Reactive oxygen species are involved in shear stress-induced intercellular adhesion molecule-1 expression in endothelial cells. Arterioscler Thromb Vasc Biol 17: 3570-3577, 1997.

11. Corson MA, James NL, Latta SE, Nerem RM, Berk BC, and Harrison DG. Phosphorylation of endothelial nitric oxide synthase in response to fluid shear stress. Circ Res 79: 984-991, 1996.

12. Davies PF. Flow-mediated endothelial mechanotransduction. Physiol Rev 75: 519-560, 1995

13. De Keulenaer GW, Chappell DC, Ishizaka N, Nerem RM, Alexander RW, and Griendling KK. Oscillatory and steady laminar shear stress differentially affect human endothelial redox state: role of a superoxideproducing NADH oxidase. Circ Res 82: 1094-1101, 1998.

14. De Vos J, Jourdan M, Tarte K, Jasmin C, and Klein B. JAK2 tyrosine kinase inhibitor tyrphostin AG490 downregulates the mitogen-activated protein kinase (MAPK) and signal transducer and activator of transcription (STAT) pathways and induces apoptosis in myeloma cells. Br J Haematol 109: 823-828, 2000.

15. García-Cardeña G, Comander J, Anderson KR, Blackman BR, and Gimbrone MA Jr. Biomechanical activation of vascular endothelium as a determinant of its functional phenotype. Proc Natl Acad Sci USA 98: 4478-4485, 2001

16. Gnant MF, Turner EM, and Alexander HR Jr. Effects of hyperthermia and tumour necrosis factor on inflammatory cytokine secretion and procoagulant activity in endothelial cells. Cytokine 12: 339-347, 2000.

17. Hernández-Rodríguez J, Segarra M, Vilardell C, Sánchez M, GarcíaMartínez A, Esteban MJ, Grau JM, Urbano-Márquez A, Colomer D, Kleinman HK, and Cid MC. Elevated production of interleukin-6 is associated with a lower incidence of disease-related ischemic events in patients with giant-cell arteritis: angiogenic activity of interleukin- 6 as a potential protective mechanism. Circulation 107: 2428-2434, 2003.

18. Hirano T. Interleukin 6 and its receptor: ten years later. Int Rev Immunol 16: $249-284,1998$
19. Hirano T, Ishihara K, and Hibi M. Roles of STAT3 in mediating the cell growth, differentiation and survival signals relayed through the IL-6 family of cytokine receptors. Oncogene 19: 2548-2556, 2000.

20. Holzinger C, Weissinger E, Zuckermann A, Imhof M, Kink F, Schöllhammer A, Kopp C, and Wolner E. Effects of interleukin-1, -2, -4, -6, interferon- $\gamma$ and granulocyte/macrophage colony stimulating factor on human vascular endothelial cells. Immunol Lett 35: 109-117, 1993.

21. Hsieh HJ, Cheng CC, Wu ST, Chiu JJ, Wung BS, and Wang DL. Increase of reactive oxygen species (ROS) in endothelial cells by shear flow and involvement of ROS in shear-induced c-fos expression. $J$ Cell Physiol 175: 156-162, 1998.

22. Irie-Sasaki J, Sasaki T, Matsumoto W, Opavsky A, Cheng M, Welstead G, Griffiths E, Krawczyk C, Richardson CD, Aitken K, Iscove N, Koretzky G, Johnson P, Liu P, Rothstein DM, and Penninger JM. CD45 is a JAK phosphatase and negatively regulates cytokine receptor signalling. Nature 409: 349-354, 2001.

23. Jalali S, Li YS, Sotoudeh M, Yuan S, Li S, Chien S, and Shyy JY. Shear stress activates p60src-Ras-MAPK signaling pathways in vascular endothelial cells. Arterioscler Thromb Vasc Biol 18: 227-234, 1998.

24. Jehle AB, Li Y, Stechschulte AC, Stechschulte DJ, and Dileepan KN. Endotoxin and mast cell granule proteases synergistically activate human coronary artery endothelial cells to generate interleukin-6 and interleukin-8. J Interferon Cytokine Res 20: 361-368, 2000.

25. Jiang GL, White CR, Stevens HY, and Frangos JA. Temporal gradients in shear stimulate osteoblastic proliferation via ERK1/2 and retinoblastoma protein. Am J Physiol Endocrinol Metab 283: E383-E389, 2002.

26. Jo H, Sipos K, Go YM, Law R, Rong J, and McDonald JM. Differential effect of shear stress on extracellular signal-regulated kinase and $\mathrm{N}$ terminal Jun kinase in endothelial cells: $G_{i 2}$ and $G \beta / \gamma$-dependent signaling pathways. J Biol Chem 272: 1395-1401, 1997.

27. Kiecolt-Glaser JK, Preacher KJ, MacCallum RC, Atkinson C, Malarkey WB, and Glaser R. Chronic stress and age-related increases in the proinflammatory cytokine IL-6. Proc Natl Acad Sci USA 100: 9090-9095, 2003

28. Levonen AL, Patel RP, Brookes P, Go YM, Jo H, Parthasarathy S, Anderson PG, and Darley-Usmar VM. Mechanisms of cell signaling by nitric oxide and peroxynitrite: from mitochondria to MAP kinases. Antioxid Redox Signal 3: 215-229, 2001.

29. Li YS, Shyy JY, Li S, Lee J, Su B, Karin M, and Chien S. The Ras-JNK pathway is involved in shear-induced gene expression. Mol Cell Biol 16: 5947-5954, 1996

30. Lin K, Hsu PP, Chen BP, Yuan S, Usami S, Shyy JY, Li YS, and Chien S. Molecular mechanism of endothelial growth arrest by laminar shear stress. Proc Natl Acad Sci USA 97: 9385-9389, 2000.

31. Maruo N, Morita I, Shirao M, and Murota S. IL-6 increases endothelial permeability in vitro. Endocrinology 131: 710-714, 1992.

32. Nakajima K, Yamanaka Y, Nakae K, Kojima H, Ichiba M, Kiuchi N, Kitaoka T, Fukada T, Hibi M, and Hirano T. A central role for Stat3 in IL-6-induced regulation of growth and differentiation in M1 leukemia cells. EMBO J 15: 3651-3658, 1996.

33. Ni CW, Hsieh HJ, Chao YJ, and Wang DL. Shear flow attenuates serum-induced STAT3 activation in endothelial cells. J Biol Chem 278: 19702-19708, 2003.

34. Ni CW, Wang DL, Lien SC, Cheng JJ, Chao YJ, and Hsieh HJ. Activation of PKC- $\epsilon$ and ERK1/2 participates in shear-induced endothelial MCP-1 expression that is repressed by nitric oxide. J Cell Physiol 195: 428-434, 2003.

35. Pedram A, Razandi M, and Levin ER. Extracellular signal-regulated protein kinase/Jun kinase cross-talk underlies vascular endothelial cell growth factor-induced endothelial cell proliferation. J Biol Chem 273: 26722-26728, 1998.

36. Ross R. Atherosclerosis: an inflammatory disease. $N$ Engl J Med 340: 115-126, 1999.

37. Schuringa JJ, van der Schaaf S, Vellenga E, Eggen BJ, and Kruijer W. LIF-induced STAT3 signaling in murine versus human embryonal carcinoma (EC) cells. Exp Cell Res 274: 119-129, 2002.

38. Sengupta TK, Talbot ES, Scherle PA, and Ivashkiv LB. Rapid inhibition of interleukin- 6 signaling and Stat 3 activation mediated by mitogenactivated protein kinases. Proc Natl Acad Sci USA 95: 11107-11112, 1998.

39. Starr R, Willson TA, Viney EM, Murray LJ, Rayner JR, Jenkins BJ, Gonda TJ, Alexander WS, Metcalf D, Nicola NA, and Hilton DJ. A 
family of cytokine-inducible inhibitors of signalling. Nature 387: $917-$ 921, 1997.

40. Surapisitchat J, Hoefen RJ, Pi X, Yoshizumi M, Yan C, and Berk BC. Fluid shear stress inhibits TNF- $\alpha$ activation of JNK but not ERK1/2 or p38 in human umbilical vein endothelial cells: inhibitory crosstalk among MAPK family members. Proc Natl Acad Sci USA 98: 6476-6481, 2001.
41. Wagner BJ, Hayes TE, Hoban CJ, and Cochran BH. The SIF binding element confers sis/PDGF inducibility onto the c-fos promoter. EMBO J 9: 4477-4484, 1990.

42. Wung BS, Cheng JJ, Chao YJ, Hsieh HJ, and Wang DL. Modulation of Ras/Raf/extracellular signal-regulated kinase pathway by reactive oxygen species is involved in cyclic strain-induced early growth response-1 gene expression in endothelial cells. Circ Res 84: 804-812, 1999.

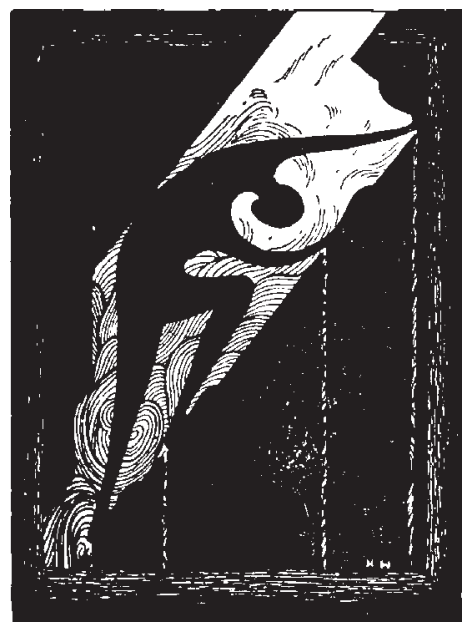

